\title{
IRACproc: A software suite for processing and analyzing Spitzer/IRAC data
}

\author{
Michael T. Schuster ${ }^{a, b}$, Massimo Marengo ${ }^{a}$ and Brian M. Patten ${ }^{a}$ \\ ${ }^{a}$ Harvard-Smithsonian Center for Astrophysics, 60 Garden St., Cambridge, MA, USA 02138 \\ ${ }^{b}$ Department of Astronomy, School of Physics and Astronomy, 116 Church Street SE, \\ University of Minnesota, Minneapolis, MN, USA 55455
}

\begin{abstract}
We have developed a post-Basic Calibrated Data pipeline processing software suite called "IRACproc". This package facilitates the co-addition of dithered or mapped Spitzer/IRAC data to make them ready for further analysis with application to a wide variety of IRAC observing programs. In acting as a wrapper for the Spitzer Science Center's MOPEX software, IRACproc improves the rejection of cosmic rays and other transients in the co-added data. In addition, IRACproc performs (optional) Point Spread Function (PSF) fitting, subtraction, and masking of saturated stars.

The under/critically sampled IRAC PSFs are subject to large variations in shape between successive frames as a result of sub-pixel shifts from dithering or telescope jitter. IRACproc improves cosmic ray and other transient rejection by using spatial derivative images to map the locations and structure of astronomical sources. By protecting sources with a metric that accounts for these large variations in the PSFs, our technique maintains the structure and photometric reliability of the PSF, while at the same time removing transients at the lowest level.

High Dynamic Range PSFs for each IRAC band were obtained by combining an unsaturated core, derived from stars in the IRAC PSF calibration project, with the wings of a number of bright stars. These PSFs have dynamic ranges of $\sim 10^{7}$ and cover the entire IRAC field of view. PSF subtraction can drastically reduce the light from a bright star outside the saturated region. For a bright star near the array center it is possible to detect faint sources as close as $\sim 15-20^{\prime \prime}$ that would otherwise be lost in the glare. In addition, PSF fitting has been shown to provide photometry accurate to $1-2 \%$ for over-exposed stars.
\end{abstract}

Keywords: Spitzer Space Telescope, InfraRed Array Camera, PSF fitting, PSF subtraction

\section{INTRODUCTION}

We have developed a collection of post-pipeline software tools for the InfraRed Array Camera ${ }^{1}$ (IRAC) on-board the Spitzer Space Telescope ${ }^{2}$ that facilitates both the co-addition of dithered/mapped data and the photometric analysis of IRAC images. Originally, this software was developed in support of the "Nearby Stars" Guaranteed Time Observer (GTO) programs; a set of interrelated programs to characterize free-floating Brown Dwarfs and to search for widely-separated sub-stellar mass companions to stars within $\sim 30 \mathrm{pc}$ of the Sun. ${ }^{3}$ These programs share a common Astronomical Observation Request (AOR - the basic Spitzer observation template) consisting of a 5-point small scale dither pattern of 30 second frame time exposures in all four IRAC bands (centered at $3.6 \mu \mathrm{m}, 4.5 \mu \mathrm{m}, 5.8 \mu \mathrm{m}$, and $8.0 \mu \mathrm{m}$ ). Some targets were also observed in high dynamic range mode to cover a wide range in brightness when photometry of the target, as well as for objects nearby on the sky, was also desired. The proximity of these systems implies that most of the program fields have a bright saturated star near the center of the array. Because the search for faint companions can be enhanced by removing the dominating glare from the saturated primary star, we were motivated to develop an integrated post-pipeline analysis system to fit and subtract saturated stars, as well as to co-add the data, identify sources and extract photometry. As such, this software package has application to a wide variety of observing programs.

Further author information: (Send correspondence to M.T.S. or M.M.)

E-mail: M.T.S.: mschuster@cfa.harvard.ed; or M.M.: mmarengo@cfa.harvard.edu 
The software suite "IRACproc"* processes IRAC data starting from the Spitzer Science Center's (SSC) Basic Calibrated Data (BCD) as provided for each AOR(s): the science BCD images, the BCD data quality (Dmask) images, and the BCD statistical uncertainty images. MOPEX, ${ }^{4,5}$ the SSC's mosaicing software, is used to co-add the BCD images based on their World Coordinate System (WCS) information taking into account geometric distortion and bad pixels. As an augmentation to MOPEX, IRACproc has two main functions: to make coaddition and other analysis of IRAC data easier, and to provide a more effective outlier rejection technique. The software also contains routines for measuring and merging photometry from the four IRAC bands. In addition, the software has routines for fitting and subtracting High Dynamic Range (HDR) Point Spread Functions (PSF).

A complicating issue with IRAC is that its images experience a flux of cosmic rays and solar protons (hereafter CR) that vary widely in size, shape, and the amount of energy deposited into the arrays. These CRs range from a single pixel, which can saturate given enough energy, to wide streaks crossing an entire array. A typical hit deposits charge in a few pixels, although some CR hits can affect many more pixels depending on the ion energy and angle of incidence (e.g. see Hora et al. $2006^{6}$ this meeting, and Patten et al. 20047). Because of the critical/under-sampled nature of the PSFs, in any single frame the CRs are often difficult to distinguish from real point-sources as well as extended sources, such as galaxies. In addition to CRs, the IRAC arrays also experience other transient or persistent effects ${ }^{8}$ including high noise pixels, hot or dead pixels, short term latents, stray light from a bright point-source off-frame, etc. To improve the rejection of these types of outliers we have implemented a technique specifically targeted for a critically and/or under-sampled camera. This method uses the spatial derivative of the images as a metric to account for changes in the PSF's projected shape on the coarse array grid that is a consequence of dithering. These PSF changes can be large and are likely to be ignored when applying common techniques such as median filtering. Using this metric for pixels covered by stars, galaxies and structured extended emission it is possible to clean IRAC images of CRs and other transients while providing the best possible photometric quality.

The remainder of this paper is organized as follows. In Section 2 we provide the foundation for using spatial derivative images as an outlier rejection metric and describe how we implement the technique for IRAC images, including our interface with MOPEX. Section 3 gives an overview of the processing and analysis steps performed by IRACproc along with some examples. In Section 4 we give a description of the IRAC HDR PSFs and a summary of our PSF fitting and subtraction technique. Finally, we conclude with suggestions on how some of our methods might be fully integrated into MOPEX or other processing software to be used in the analysis of a wide variety of IRAC observing programs.

\section{OUTLIER REJECTION IN UNDER-SAMPLED IMAGES}

One of our goals was to develop a technique to remove outliers from IRAC images that is more effective than common outlier rejection schemes, such as median filtering or sigma-clipping. The method we implemented uses the spatial derivative of the images to estimate changes in the coarsely sampled PSF shape that is a consequence of dithering or telescope jitter. Specifically, the method does not unnecessarily reduce the number of pixels to be co-added at each sky location (the depth of coverage), nor leave true outliers untouched. A variation of this technique was first used in the Space Telescope Science Institute's DITHER data reduction package for Hubble Space Telescope images ${ }^{9}$ to mitigate the effects of interpolation in outlier rejection. This method is general enough that it can be adopted for any camera whose Point Spread Function is under and/or critically sampled, as is IRAC's.

In any critically or under-sampled image the apparent shape of the PSF, the Pixel Response Function (PRF), depends on its projection onto the pixel grid. Slight sub-pixel shifts of the array, from dithering or telescope jitter, will result in changes to this projection for sharply peaked point-sources. For example, sampling the continuous PSF with relatively large pixels and then dithering by small amounts will cause objects to appear to have very different profiles between frames. For an example of these PRF changes see Figure 1. These PRF variations are significantly larger than expected from statistical fluctuations alone, and can be estimated by measuring the difference between adjacent pixels in the PRF. The actual temporal change in pixel value is a combination of this effect between frames and other sources of noise. However, it is still necessary to reject an outlier on top

* IRACproc runs within PDL, a numeric extension for Perl available from pdl.perl.org. 
IRAC [3.6] PRF Changes - Vertical Cut

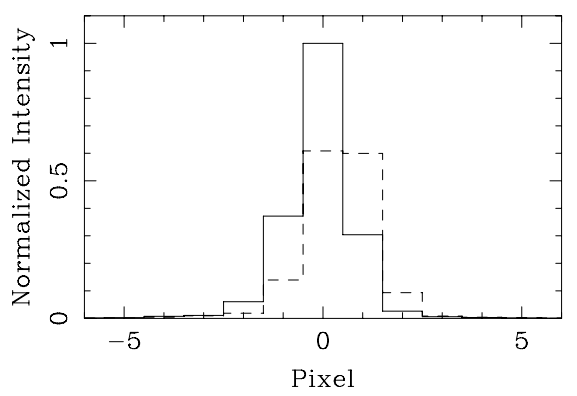

IRAC [5.8] PRF Changes - Vertical Cut

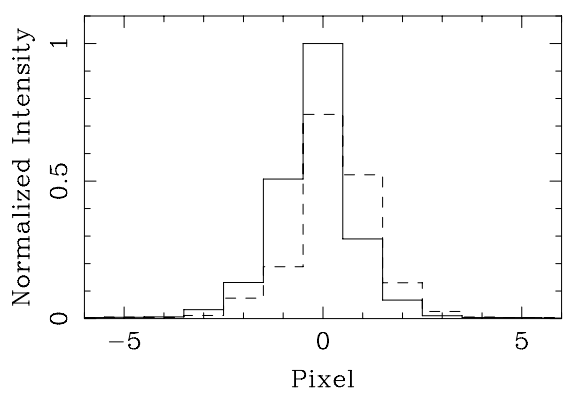

IRAC [4.5] PRF Changes - Vertical Cut

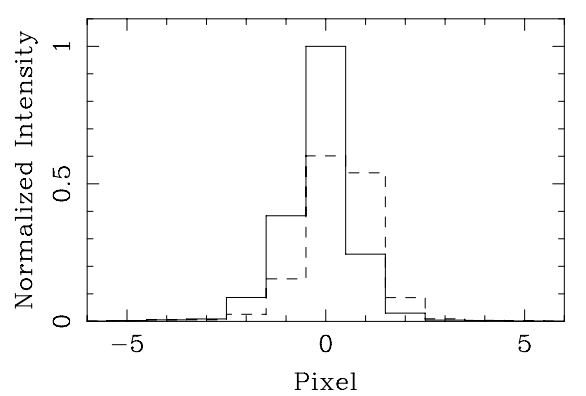

IRAC [8.0] PRF Changes - Vertical Cut

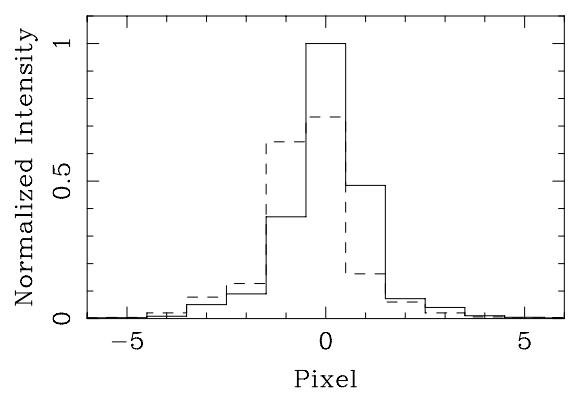

Figure 1. Cuts across Pixel Response Functions (PRF) in each of the four IRAC bands. Solid: PSF with peak centered close to the middle of a pixel. Dashed: PSF with peak centered close to the corner of a pixel (peak flux now spread among 3 additional pixels). The peak can increase in value from one frame to the next by as much as $64 \%$ at $3.6 \mu \mathrm{m}, 66 \%$ at $4.5 \mu \mathrm{m}, 35 \%$ at $5.8 \mu \mathrm{m}$, and $56 \%$ at $8.0 \mu \mathrm{m}$.

of a point-source when it changes the value by more than what can be expected from shifting the image by a fraction of a pixel. The local spatial derivative, defined as the difference between adjacent pixels, is one method that can gauge the reliability of a pixel's value on-source. This rejection criterion protects the PRF shape while identifying true outliers, and is valid for any set of under and/or critically sampled images.

Outlier rejection methods that use only the statistical information of a pixel stack, such as median filtering or sigma-clipping, are incapable of distinguishing true outliers from those values susceptible to PRF changes. These methods have no means by which to recognize as real the relatively large variations in pixel value that are a result of the coarse PSF sampling, information that can be recovered only if the values of neighboring pixels are taken into account. In the case of sigma-clipping, depending on the value chosen for the clipping threshold, statistical methods will either ignore outliers or will erroneously reject the PRF peaks. The effect in the final image is two-fold: (1) the shape of the PSF is altered, and (2) the photometry measured from that altered PSF is no longer as reliable. See Section 2.2 for a quantitative discussion.

\subsection{A Robust Outlier Rejection Technique}

Robust outlier rejection for any data set requires two pieces of information: (1) a "best estimate" of the expected value in the pixel stack, and (2) a metric to decide which values in the stack are real data from a source or an outlier unrelated to the source. Our method for outlier rejection is a form of sigma-clipping, with the mean or median replaced with the best estimate and the "sigma" replaced with the metric.

As an approximation to the actual mosaic, the best estimate image provides a reasonable basis for deciding which values are outliers. IRAC observers are encouraged to dither or repeat their observations in order to have more than one frame, and pixel, at each sky location. Since it is unlikely an outlier will appear in all frames at the same sky location, this strategy generally makes it possible to derive a reasonable estimate of the actual flux at 
that sky position by selecting the most likely value in the pixel stack. A best estimate image should be free of outliers, while preserving the underlying structure of the noise and sources in the image.

The best estimate image can be constructed in different ways according to the number of frames covering each piece of sky, that is, the coverage depth. With a relatively high depth of coverage ( $\sim 5$ or more overlapping frames), the median image can be used as a best estimate image. Because most outliers have large positive values in astronomical data, it is possible to improve upon the median by instead choosing a value in the pixel stack that is adjacent to the median. Typically this shift, or bias, is towards lower values and has the effect of mitigating outliers even when as much as half of the data is bad (e.g. CRs), making it a more robust choice for the best estimate. This so-called "biased median" is defined as the value in the stack that is the $n^{\text {th }}$ closest neighbor above, or below the median, where the bias is $n$.

For shallow coverage depths ( $2-5$ overlapping frames), a biased median is not practical since a bias by one value towards the minimum will always select very low values for the best estimate. In this case, we introduce the concept of the "absolute minimum deviation" (AMD) image as a very reliable best estimate designed to find the pixels closest to the most likely value. The AMD image is calculated in the following way:

$$
A M D(x, y)=\min _{s=1 . . N_{c o v}(x, y)}\left\{\left|I_{B C D}^{(s)}(x, y)-I_{p e d}(x, y)\right|\right\}
$$

where $s$ is the index for the pixel stack, $N_{\text {cov }}$ is the coverage depth, $I_{B C D}^{(s)}$ is the pixel value from the $s^{t h}$ frame, and $I_{\text {ped }}$ is the background pedestal in all of the frames. The AMD image is a mosaic of frames whose pixels are closest to the background pedestal in absolute value. The global background for all frames is determined from another simpler best estimate image, and is typically the mode of a median image. It is essential that a single pedestal value (or image) is used, as opposed to background subtracting the input frame individually, since any large scale background structure may yield different pedestals for overlapping frames. Subtracting different background values for each frame would make it harder to correctly choose the best estimate by introducing new pedestal off-sets. Conversely, without background subtraction the AMD would effectively select the minimum value in most cases, instead of the value closest to the background pedestal. Thus, for shallow coverage depths the AMD image improves the choice for the best estimate in the background, while providing nearly the same value on-source as a median biased towards lower values.

For a metric, IRACproc uses the "maximum local derivative" (MLD) image, defined as the maximum of the absolute differences between each pixel of the best estimate image and its four closest neighbors. ${ }^{9}$

$$
M L D(x, y)=\max _{i=1 . .4}\left\{\left|I_{b e}(x, y)-I_{b e}(x, y ; i)\right|\right\}
$$

where $i$ is the index of the neighboring pixels, $I_{b e}$ is the value in the best estimate image, and $I_{b e}(x, y ; i)$ is the value of the neighboring pixel. Since the MLD image is a spatial derivative of the best estimate image, by construction it can account for the basic structure of the PRF and the noise of the background in the co-added image. However, since the noise in the best estimate image scales as the square root of the coverage depth, it returns a metric in the background areas that is much smaller than the noise in the individual frames, and as such it does not represent the best metric for the background. Therefore, a robust methodology for determining the metric will choose, on a pixel-by-pixel basis, the largest value between the MLD and a more reliable metric for the background (such as the MAD - Median Absolute Deviation from the median, see MOPEX documentation ${ }^{5}$ ).

With these best estimate and metric images, we can reject as outliers pixels that satisfy the following condition:

$$
\left|I_{B C D}(x, y)-I_{b e}(x, y)\right|>\operatorname{scale} \times \operatorname{metric}(x, y),
$$

where $I_{B C D}$ is the value in the $\mathrm{BCD}$ frame, $I_{b e}$ is the value in the best estimate image, and scale is the threshold scale factor for sigma-clipping. The final mosaic is an average of the remaining "good" values in the input pixel stack. 


\subsection{Quantitative Effects for IRAC Photometry}

The large variations in the IRAC Pixel Response Functions, illustrated in Figure 1, imply that it will be difficult to reject real outliers when they occur on the peaks of point-sources. It is useful to analyze how significant of an effect this can be when using the technique described in the previous section to protect "real" data from being rejected.

As an example, consider a hypothetical CR that covers one pixel and lands on the peak pixel of a point-source. In each of the four IRAC bands any $\mathrm{CR}$ with a value less than the difference in peak values seen in Figure 1 should not be rejected. Using the smallest aperture recommended in the IRAC Data Handbook ${ }^{8}$ (radius of 2 native IRAC pixels) we can estimate the maximum effect on the measured flux of the source. In this case, estimates of the worst CR that must be let through would be $10.6 \%$ at $3.6 \mu \mathrm{m}, 11.5 \%$ at $4.5 \mu \mathrm{m}, 5.4 \%$ at $5.8 \mu \mathrm{m}$, and $6.4 \%$ at $8.0 \mu \mathrm{m}$, as a percentage of the total point-source flux in a single BCD frame. It is unlikely to have a CR on top of a source in multiple frames, and averaging the pixels from all frames implies that the final effect on photometry would be to divide each the above estimates by the coverage depth. With as few as 5 frames of coverage, the maximum contribution this hypothetical CR would produce is $1-2 \%$, approximately the photometric accuracy of the IRAC camera. For CRs that cover more than one pixel the rejection level is lower for those pixels that are adjacent to the peak of the point-source, and very low for background areas. This indicates that the contributions from low-level CRs that slip through with our outlier rejection technique rapidly become very small, because the metric is well adapted to the structure of the data.

By comparison, using median filtering, a least squares fit, or a sigma-weighted sum to produce a clean image will preferentially ignore frames with large PRF variations. Additionally, using sigma-clipping with a statistically determined metric (such as the error mean or even the MAD) on-source will either: a) reject both good and bad values on-source while cleaning the background areas of the image with a low rejection threshold, or b) allow low-level CRs to pass through at the expense of a very polluted background with a high rejection threshold. The former case manifests itself as a reduction in coverage depth on-source, and the latter case produces images that still have many low-level outliers, including the edges of the larger and higher-strength CRs (i.e. a CR "doughnut").

This example also provides an upper-limit error estimate for the photometry when using the common image processing methods mentioned above on IRAC images. If we consider a mosaic of $N_{\text {total }}$ co-added frames, with $N_{\text {high }}$ point-source peaks erroneously rejected/ignored as the result of large PRF variations, then the photometry would be affected by $\sim\left(N_{\text {high }} / N_{\text {total }}\right) \times\left(I_{\text {peak }}^{\text {center }}-I_{\text {peak }}^{\text {corner }}\right) / F_{\text {total }}$ * . Having a large number of frames $\left(N_{\text {total }}\right)$ does not diminish this effect, since there will always be a fraction of the frames with sharply peaked PRFs that is determined by the probability of a point-source peak landing in the middle of a pixel as compared to near an edge. Thus, the rejection of peak PRF pixels could potentially affect many sources, and in the worst case, affect their photometry up to $\sim 11 \%$ at $3.6 \mu \mathrm{m}$ and $4.5 \mu \mathrm{m}$, and $\sim 6 \%$ at $5.8 \mu \mathrm{m}$ and $8.0 \mu \mathrm{m}$.

If the methods described in this paper are used to maintain the coverage depth and photometric fidelity, it is possible to have the best of all scenarios; accurate photometry, effective rejection of outliers, and a clean background.

\subsection{Implementation}

To produce mosaics clean of CRs and other transient effects, IRACproc uses two layers of outlier rejection. The first layer uses the AMD image as the best estimate and is the only method effective for a coverage depth of two frames. This layer projects the AMD image onto each BCD frame and rejects outliers on a frame by frame basis. As we will discuss in this section, this layer has problems rejecting low-level outliers on sources in higher-coverage areas of the mosaic. The second layer is very effective for higher-coverage depths ( $>5$ frames). This layer projects each BCD frame onto the final grid and rejects outliers using a median image as the best estimate. For moderate coverage depths (2-4 frames), or mixed coverage depths (areas with high coverage mixed with areas of low coverage) the combined effect of both layers effectively cleans the mosaic of transients.

\footnotetext{
${ }^{*}$ For a median image this effect is approximately $\mid\left\langle I_{\text {peak }}>-\operatorname{median}\left(I_{\text {peak }}\right)\right| / F_{\text {total }}$
} 


\subsubsection{Outlier rejection: first layer}

This first layer detects outliers in each BCD frame and records their locations by modifying the BCD data quality (Dmask) images. The flagged outliers are masked during co-addition.

As mentioned before, the MLD images are not a good estimate of the noise in the background of the individual BCD frames, since they scale as the square root of the coverage depth. In addition, the MLD will slightly underestimate the value of the derivative, since it is derived from the AMD image which effectively selects the minimum value on-source. To correct these problems and provide a good metric in the background for all coverage depths, we create "effective derivative" images by multiplying the MLD by the square root of the coverage image $N_{\text {cov }}(x, y)$. As the coverage depth varies significantly across a mosaic, outlier identification remains robust on the background. However, since this correction also increases the metric on-source in high-coverage areas it prevents detection of low-level outliers in this first layer. Because the best estimate and metric images are also projected back to the grid of the original input frames using some form of interpolation, there is an additional loss of precision in the outlier rejection. These limitations are balanced by the second layer of outlier detection which is discussed in Section 2.3.2.

To flag outliers we clear the BCD Dmask "radhit" bit (bit 9), and reset it if the pixel is determined to be an outlier:

$$
\left|I_{B C D}(x, y)-I_{p e d}(x, y)-A M D_{B C D}(x, y)\right|>C R \_T H R E S H \times M L D_{B C D}(x, y) \times \sqrt{N_{c o v}(x, y)},
$$

where $I_{B C D}$ is the value in the BCD frame, $I_{p e d}$ is the background pedestal, $\mathrm{AMD}_{B C D}$ and $\mathrm{MLD}_{B C D}$ are the projection onto the BCD frame, and CR_THRESH is the outlier rejection threshold scale.

\subsubsection{Outlier rejection: second layer}

IRACproc acts as a wrapper for the SSC's co-addition software MOPEX. The second layer of outlier rejection is performed by MOPEX's multi-frame temporal outlier rejection using either an internally computed sigma* ${ }^{*}$ the BCD statistical uncertainty images as an alternative. IRACproc enhances outlier rejection within MOPEX by replacing the BCD uncertainty images with our own metric images. ${ }^{\dagger}$ As discussed in Section 2.1, this choice ensures that a reliable metric is being used for the rejection of true outliers.

The median image is used as the best estimate and the MLD image, combined with the BCD uncertainty image $(B U N C)$, is used as the external metric. By combining the BCD uncertainty image, with an appropriate weight, to the MLD image, the metric is effectively raised in the background. This formulation simultaneously protects the depth of coverage for point-sources while aggressively removing outliers on or near them, and very aggressively cleans the background of outliers.

The metric images are calculated with the following formula:

$$
\begin{array}{r}
\text { metric }_{B C D}=\left(1-B U N C_{\_} F R A C\right) \times D E R I V_{-} S C A L E \times M L D_{B C D} \\
+B U N_{-} F R A C \times B U N_{\_} S C A L E \times B U N C,
\end{array}
$$

where the final metric image is projected onto the $\mathrm{BCD}$ frame preserving a close approximation to the refined WCS used with Spitzer images, MLD $_{B C D}$ (derived from the median mosaic) is projected onto the BCD frame. The parameters DERIV_SCALE and BUNC_SCALE scale the MLD image and the BCD uncertainty image independently. By selecting a non-zero value for the option $B U N C \_F R A C$, the BCD uncertainty images can be included in the metric images, and setting $B U N C_{-} F R A C$ to 1 will result in the metric images including only the BCD uncertainty images.

\footnotetext{
${ }^{*}$ Currently (v030106), this internal sigma is the MAD - Median Absolute Deviation from the median, see MOPEX documentation. ${ }^{5}$ Previously, it was the error on the mean.

${ }^{\dagger}$ As IRACproc has evolved it has consistently improved outlier rejection within MOPEX with this replacement. IRACproc v3.1 and earlier used only the MLD. IRACproc v3.2-3.3 excluded the BCD uncertainty images for coverage depths of 5 or less to prevent the increased photon noise associated with CRs from affecting the weighted average metric.
} 


\section{PROCESSING IRAC DATA WITH IRACPROC}

Creating mosaics with IRACproc is a 4 -step process.

1. The BCD data are organized into a modular directory structure that separates the software configuration files, intermediary products and final mosaics. For sub-array data, there is a routine for splitting each 64-layer image cube into individual FITS images for further processing.

2. Images are selected for processing based on: channel number, exposure time, WCS coordinates, or on/off field frames, for example. The routine that automates this selection process also prepares the parameter files that control MOPEX.

3. Additional outside processing can be performed, including cosmetic alterations for muxbleed, banding, and column pull-down, or subtraction of a specific background.

4. With the data prepared, the mosaics are created for each IRAC band with a single routine. In brief, this routine registers the background levels of each input frame using the MOPEX overlap correction module. A median image is created and used to calculate the background pedestal for the AMD image described in Section 2.3.1. The first layer of outlier rejection is then performed, followed by the creation of the metric images for the second layer, outlined in Section 2.3.2. Once these preliminary steps are completed, the final mosaic with outliers rejected is created by MOPEX (See MOPEX documentation ${ }^{5}$ for directory structures and output).

IRACproc also includes a set of scripts to perform some basic analysis of the processed data. There are routines to copy images or image sections, subtract a background pedestal level, and/or convert IRAC image units from MJy/sr ( $10^{6} \mathrm{Jy}$ per steradian) to Jy, DN/sec, DN, or $e^{-}$. Additionally, there is an IRAF* script to detect sources and perform aperture photometry using the APPHOT package. This script was originally developed in support of the Spitzer/IRAC "Nearby Stars" GTO programs, ${ }^{3}$ but in fact can be applied to any IRAC photometry program. A stand alone perl script merges photometry from multiple wavelength bands by correlating magnitudes based on the WCS coordinates of the sources. Using these scripts we were able to efficiently analyze the multi-band photometry for hundreds of sources in each field of the "Nearby Stars" programs.

As an example of how well IRACproc can work with the depth of coverage limited to just two frames, Figure 2 shows the images before and after outlier rejection. The mosaics on the left are raw in the sense that no outlier rejection has been performed on them (known bad pixels have been masked). The mosaics in the middle are the cleaned IRACproc images, followed by the coverage maps on the right. In this example the frame time is $30 \mathrm{sec}$. The cleaned mosaics and their accompanying coverage maps show that the first layer of outlier rejection (the only method used here) is effective at removing all types of CRs, even those directly on sources. For example, look for the large CRs in the [3.6] and [5.8] bands, and the coverage maps on the right. In addition, Figure 3 shows another example with a depth of coverage equal to 5 frames (with small scale random dither pattern).

\section{IRAC HIGH-DYNAMIC RANGE POINT SPREAD FUNCTION SUBTRACTION}

IRACproc also provides a framework for easy PSF subtraction, to allow the suppression of the light from bright sources even in cases of severe saturation. This facility was designed to allow PSF subtraction for the bright stars in the "Nearby Stars" GTO programs, needed to search for possible fainter companions within the IRAC field of view. An example of "before and after" PSF subtraction is given in Figure 3 for the nearby star GJ 832. The top left panel shows the IRAC "raw" $4.5 \mu \mathrm{m}$ mosaic of the star (combining 5 frames, 30 seconds each, without outlier rejection); note how a large fraction of the image is covered by the extended features of the IRAC PSF. In the bottom left panel the IRAC PSF has been removed from the final IRACproc mosaic, revealing faint sources as close as $\sim 15-20^{\prime \prime}$ from the star (inside that radius, saturation and high photon noise prevents an accurate

\footnotetext{
${ }^{*}$ Image Reduction and Analysis Facility. IRAF is distributed by the National Optical Astronomy Observatories, which are operated by the Association of Universities for Research in Astronomy, Inc., under cooperative agreement with the National Science Foundation. Available from iraf.noao.edu.
} 

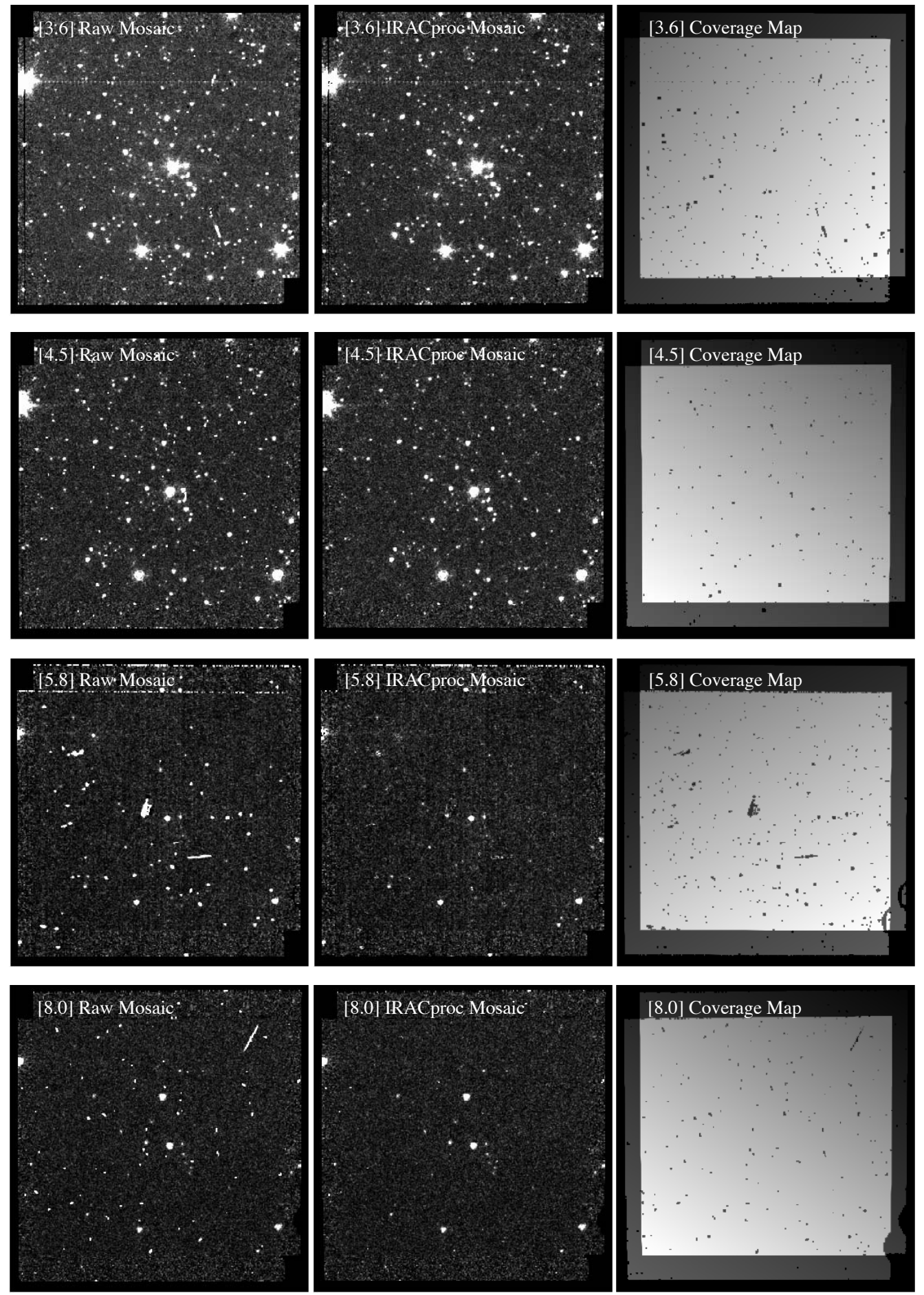

Figure 2. Example of IRACproc mosaics with a coverage depth of 2 for each IRAC band. Left: raw mosaic with bad pixels masked. Middle: IRACproc mosaic. Right: coverage map, the number of frames co-added for each pixel. These images are from AOR 3938304, PID 35, frame time 30 sec. 
PSF subtraction). The IRACproc coverage map (top right) shows that our software is effective in removing CRs and other outliers even in presence of high signal from the extended PSF. The last panel shows a custom mask superposed on the PSF-subtracted image in order to cover the high PSF-subtraction residuals that would interfere with source detection while deriving the photometry of the sources in the field. The application of the mask is also a feature provided by the IRACproc PSF-subtraction package.

The PSFs used by IRACproc were derived by combining the images of several very bright stars in the "Spitzer Fabulous Four Debris Disk Stars" GTO program (PID 90), plus some fainter stars observed with the specific purpose of measuring the IRAC PSF. ${ }^{10}$ The "Fabulous Four" program consists of the observation with all Spitzer instruments of the original four young main sequence stars found by IRAS to have a "second generation" (or "debris") protoplanetary disk. These stars (Vega, $\beta$ Pictoris, Fomalhaut and $\epsilon$ Eridani, plus the reference star $\epsilon$ Indi) have been observed with IRAC using a very long total exposure (up to 1 hour), in order to search for planetary mass companions within the IRAC field of view. With the exception of $\beta$ Pictoris, which is indeed extended at IRAC wavelengths, the debris disks are not detected by IRAC for the other stars. The data obtained with such long integration, albeit saturated in the core, thus provide very high $\mathrm{S} / \mathrm{N}$ images of the extended parts (the faint extended tail and the diffraction spikes) of the IRAC PSF. By merging these "extended PSFs" with unsaturated cores from fainter reference stars, using a semi-transparent mask to ensure a smooth transition, we have created a set of High Dynamic Range (HDR) PSFs for IRAC. These final HDR PSFs are normalized in surface brightness units (in MJy/sr, the same as the BCD) to have the total flux of a zero magnitude A-type star (Vega, which was indeed part of the "Fabulous Four" stars). The relative normalization between the core and extended components of the PSF is accurate to the level of the IRAC absolute photometric calibration (better than $10 \%$ ). The final S/N of the HDR PSFs is $\sim 10^{7}$ for the 3.6 and $4.5 \mu \mathrm{m}$ IRAC bands, and $\sim 10^{6}$ for 5.8 and $8.0 \mu \mathrm{m}$.

The shape of the PSF depends on its position on the IRAC array: the diffraction spikes and other features shift and change in relative strength with respect to the core, and the core itself changes in shape as the point source moves around the array. Inter-pixel phase and intra-pixel sensitivity also affect the PRF shape at all positions on the arrays. As a consequence, since the stars used to create the HDR PSFs have been observed at the center of the IRAC arrays, the best PSF subtractions are obtained when the stars to be subtracted are also observed in the same position, and with the same dither pattern (36 points Reuleaux, small scale). Due to nonlinear components in the IRAC PSF (e.g. the "electronic artifacts" described in the IRAC Data Handbook ${ }^{8}$ and in Marengo et al. 2006, ${ }^{11}$ among them the so-called "banding", "muxbleed" and "column pull-down"), and a "charge-diffusion" in the PSF cores at $3.6 \mu \mathrm{m}$, the best PSF subtraction is obtained for point sources with similar magnitude of the stars used to create the HDR PSFs. To obtain the best PSF subtraction for non-saturated stars, stars brighter than Vega, or stars observed far from the center of the IRAC arrays, it is thus better to allow for the observation of a reference star having similar brightness to the science target, observed with the same dither pattern and in the same area of the IRAC arrays.

We have tested the accuracy of the PSF subtraction provided by IRACproc by measuring both PSF fitting and aperture photometry of 6 M5.5 - M9 stars observed with the IRAC HDR mode, for which two exposures (one unsaturated of $1.2 \mathrm{sec}$, and one saturated of $30 \mathrm{sec}$ ) were available. By measuring the scaling factor between the saturated images and the HDR PSF we have then measured the brightness of each of our test stars, and found that the derived magnitudes match within $\sim 1-2 \%$ of the aperture photometry obtained on the unsaturated short exposure frames.

The HDR PSFs distributed with IRACproc are available with three pixel scales: $0 .{ }^{\prime \prime} 3$, $0 .{ }^{\prime \prime} 4$, and $1 .{ }^{\prime \prime} 22 / \sqrt{2}$ (0." 8626716) per pixel. Larger sampling can be achieved by re-binning the $0 . .^{\prime \prime} 3 /$ pix or $0 . .^{\prime \prime} 4 /$ pix PSFs. The PSF images are approximately $312^{\prime \prime} \times 312^{\prime \prime}$, the size of the IRAC arrays.

\section{CONCLUSIONS}

IRACproc is a software suite designed to process and analyze IRAC data. It uses the SSC's post-BCD processing software MOPEX for co-addition and significantly improves outlier rejection with spatial derivative images. Included with IRACproc is a set of IRAC High Dynamic Range Point Spread Functions which can be used to effectively remove the dominating light from a bright star to reveal faint sources. 

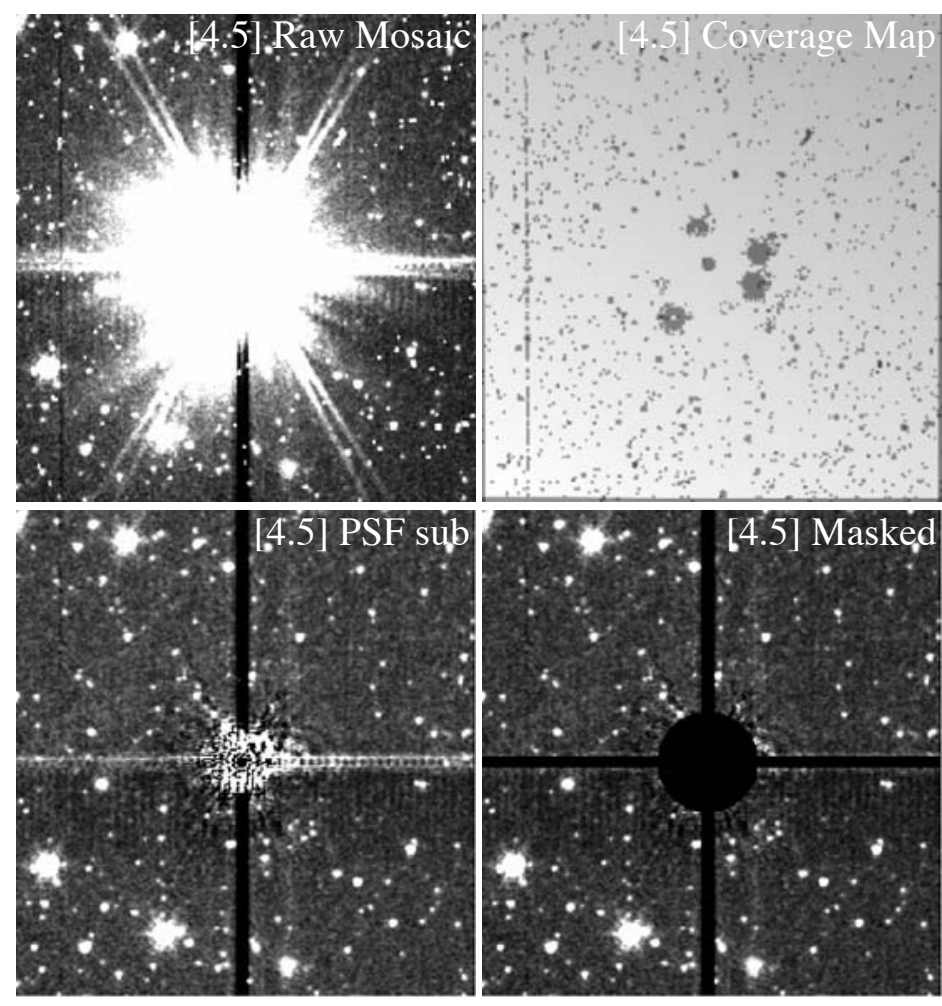

Figure 3. Spitzer/IRAC $4.5 \mu \mathrm{m}$ image of a typical bright $\operatorname{star}\left(\operatorname{mag}_{[4.5]}=4.25\right)$. Top left: raw mosaic with bad pixels masked. Top right: coverage map, the number of frames co-added for each pixel. Bottom left: PSF-subtracted IRACproc mosaic. Bottom right: mosaic with PSF subtraction residuals masked. With PSF subtraction it is possible to identify sources as close as $\sim 15-20^{\prime \prime}$ from the primary. Note that the short term latents from the bright star are masked in the final mosaic. This image is from AOR 3914240, PID 33, frame time $30 \mathrm{sec} \times 5$.

The key to our outlier rejection method is a robust choice for a best estimate and metric for outlier rejection. MOPEX already contains much of the underlying infrastructure needed to generate the biased median, absolute minimum deviation, and maximum local derivative images described in Section 2.1. The internally computed sigma within MOPEX (the Median Absolute Deviation from the median) is a good metric for the background, and even on the wings of point-sources. However, it is still not completely effective at taking into account the large fluctuations in the IRAC Pixel Response Functions and has difficulty identifying real outliers on sources.

Since MOPEX already chooses the maximum available sigma for temporal outlier rejection, it could calculate the maximum local derivative of the median image and directly compare those values to the internal sigma. With this implementation, MOPEX could have an extremely robust and efficient outlier rejection scheme for any coverage depth.

\section{ACKNOWLEDGMENTS}

We thank David Makovoz for adding the absolute minimum mosaic as an option for the MOPEX mosaic module specifically for IRACproc. This work is based in part on observations made with the Spitzer Space Telescope, which is operated by the Jet Propulsion Laboratory, California Institute of Technology under NASA contract 1407. Support for this work was provided by NASA through subcontract 2-1062296 issued by JPL/CalTech. 


\section{REFERENCES}

1. G. Fazio et al., "The Infrared Array Camera (IRAC) for the Spitzer Space Telescope," Astrophysical Journal Supplement Series 154, pp. 10-17, 2004.

2. M. Werner et al., "The Spitzer Space Telescope Mission," Astrophsical Journal Supplement Series 154, pp. 1-9, 2004.

3. B. M. Patten et al., "A Search for Widely Separated Sub-Stellar Mass Companions to Nearby Stars with Spitzer/IRAC," in Protostars and Planets V, Conf. Proc. LPI Contrib. No. 1286, p. 8042, 2005.

4. D. Makovoz and I. Khan, "Mosaicking with MOPEX," in Astronomical Data Analysis Software and Systems VI, P. L. Shopbell, M. C. Britton, and R. Ebert, eds., ASP Conf. 132, 2005.

5. D. Makovoz, Spitzer Mosaicker, SSC California Institute of Technology, California, 2006 (Version 1.9). ssc.spitzer.caltech.edu/postbcd/documentation.html.

6. J. L. Hora, B. M. Patten, G. G. Fazio, and W. Glaccum, "The effects of cosmic rays and solar flares on the IRAC detectors: the first two years of in-flight operation," in Astronomical Telescopes and Instrumentation, Proc. SPIE 6276-21, 2006.

7. B. M. Patten, J. L. Hora, G. G. Fazio, P. Barmby, Z. Wang, and D. Makovoz, "Normal and unusual transient events in IRAC images," in Astronomical Telescopes and Instrumentation, Proc. SPIE 5487-91, 2004.

8. W. T. Reach et al., Infrared Array Camera Data Handbook, SSC California Institute of Technology, California, 2006 (Version 3.0).

9. A. M. Koekemoer et al., HST Dither Handbook, STScI, Baltimore, 2000 (Version 1.0).

10. M. Marengo et al., in preparation, 2006.

11. M. Marengo, S. T. Megeath, G. G. Fazio, K. R. Stapelfeldt, M. W. Werner, and D. E. Backman, "A Spitzer/IRAC Search for Substellar Companions of the Debris Disk Star $\epsilon$ Eridani," Astrophsycial Journal in press, 2006. 